\section{Behavioral Economics and the Future of Biosimilars}

\author{
Chadi Nabhan, MD, MBA, and Bruce A. Feinberg, DO
}

The expanded use of biologic agents in cancer has contributed substantially to the continued rise in US healthcare costs. Recent statistics show biologics accounting for $62 \%$ of the $\$ 18.5$ billion USD total Medicare Part B drug spending, exerting additional pressure for drug-savings measures. ${ }^{1}$ Some experts have proposed that biosimilars could mitigate this continued upslope through competing market prices relative to their reference product. This is especially relevant because many patents for biologics are expiring within the next 5 years, including those for 4 of the top 10 drugs by cost. ${ }^{2}$ Such bending of the cost curve would require robust acceptance of biosimilars by the prescribing oncologists and the patients they treat.

Biosimilars contain a highly similar version of the active substance of an already approved biologic agent, referred to as the "reference product." Regulatory agencies such as the FDA allow at least 1 of the approved indications for the reference product to be listed as an indication for the biosimilar, but the FDA mandates that safety, efficacy, dosing, route of administration, and immunogenicity are established first. ${ }^{3}$ In addition, the FDA allows extrapolation of safety and efficacy data from one biosimilar indication to another after rigorous requirements are fulfilled. ${ }^{4}$ These requirements are meant to assure clinicians that extrapolation is safe and effective. Extrapolation is a cost-saving measure welcomed in a resource-constrained environment that recognizes limitations of conducting expensive confirmatory randomized studies for every indication in every disease stage. ${ }^{4}$

Behavioral economics is a discipline that combines insights from psychology, economics, judgment, and decision-making to better understand, predict, and potentially change human behavior. Tversky and Khaneman ${ }^{5}$ launched the field with a series of experiments that confirmed that people are often irrational economic actors. The authors proposed that such irrational behavior relied on a limited number of heuristic principles, which reduce the complex intellectual tasks of assessing probabilities and predicting values to simplified judgment operations. These heuristics, however, can lead to serious erroneous biases in decision-making that may have significant impact on healthcare reform.

\section{Behavioral Economics of Oncologists' Prescribing}

Biosimilars are entering healthcare markets at a time when fee-for-service reimbursement methodologies are rapidly being replaced with value-based care models in which prescribers share financial risk. These new reimbursement models challenge our understanding of physicians' decision-making and prescribing preferences. The proposed dissolution of simplistic rational economics (ie, the more you do or prescribe, the more you are paid) and replacement with complex models that compare clinical and financial outcomes of providers with those of both historical benchmarks and providers' peers may have broad consequences on physician prescribing.

If providers were rational economic actors, then biosimilar adoption could play a significant role in such financial outcome models given projections of $( \pm 30 \%)$ cost differences with their reference brands. ${ }^{2}$ Behavioral economics healthcare research, albeit limited, suggests that neither patients nor providers are rational economic actors due to numerous inherent biases in their decision-making. ${ }^{6}$ Researchers have be-

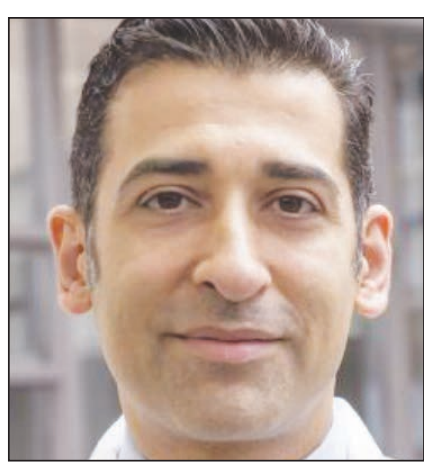

Chadi Nabhan, MD, MBA

Dr. Nabhan is a Vice President and Chief Medical Officer of Cardinal Health Specialty Solutions, a division of the biopharmaceutical segment of Cardinal Health. He works across all business units of specialty solutions providing resources to pharmaceutical companies, providers, payers, patients, and policy makers.

Dr. Nabhan is heavily engaged in data analytics, strategic initiatives, regulatory, and logistical initiatives in a value-based environment and a rapidly changing landscape of healthcare delivery. He is interested in developing strategies to improve cancer care delivery in an era where reimbursement models are changing. His focus is on the business and economic aspect of oncology as they impact all stakeholders.

Dr. Nabhan is a hematologist and medical oncologist with special interests in malignant hematology (leukemias and lymphomas) and genitourinary malignancies. He has been the primary investigator on many clinical trials, and has authored more than 200 peerreviewed articles, abstracts, or book chapters.

Prior to joining Cardinal Health Dr. Nabhan was the Medical Director of the Clinical Cancer Center at the University of Chicago overseeing the clinical operations of cancer clinics. He was instrumental in obtaining the QOPI certification for the University of Chicago and an integral member of the team implementing clinical care pathways.

doi:10.6004/jnccn.2017.7023

The ideas and viewpoints expressed in this editorial are those of the author and do not necessarily represent any policy, position, or program of NCCN. 


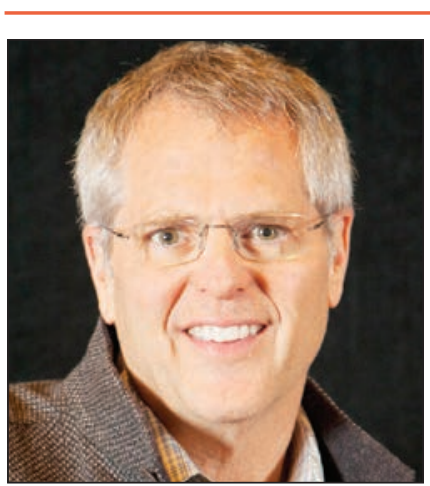

Bruce A. Feinberg, DO

Bruce A. Feinberg, DO, serves as Vice President of Clinical Affairs and Chief Medical Officer for Cardinal Health Specialty Solutions. He works across the specialty solutions businesses, consulting with payers, providers, pharma, patients, and policy makers. Dr. Feinberg has played a vital role in the nascent valuebased healthcare movement and was instrumental in developing PathWare, leading decision support and transaction software. Since joining Cardinal Health, Dr. Feinberg has made more than 70 peer-reviewed contributions to value-based healthcare literature. In 2015, Dr. Feinberg was honored as a PharmaVoice 100 most inspirational life-science leaders.

Dr. Feinberg is the author of bestselling books and has been consulted by prominent national media outlets, including The New York Times, Wall Street Journal, Forbes, and CNN. As host of The Weekly Check-Up on WSB Radio in Atlanta, he provides the community the latest health information and an opportunity to express their views on health and medicine.

Prior to joining Cardinal Health, Dr. Feinberg was the founder and CEO of Georgia Cancer Specialists (GCS), one of the first and largest integrated oncology specialty practices in the United States. At GCS, he pioneered health information technology, the pathways movement, and the oncology medical home, publishing the first report of their impact on financial and clinical outcomes in 1998. gun to address the impact of these biases on physician behavior in regard to their compensation, referencing behavioral economics theories and human factors in the design of the Affordable Care Act (ACA) and the American Health Care Act of 2017 (AHCA). ${ }^{7}$ Biosimilars may represent a special case in which understanding the biases impacting physician prescribing will be critical to predicting the rate and extent of their adoption and the related impact on market economics. We explore 7 biases in physician decision-making that may significantly impact biosimilar adoption.

\section{Loss Aversion}

Loss aversion bias highlights how fear of monetary loss can dominate decision-making. Research shows that losses have about twice the psychological impact of commensurate gains. ${ }^{6}$ Current reimbursement models for infused biologics reimburse the provider a fixed margin above the cost of the drug. If biosimilars have a lower price point, then they will generate a lower margin. The loss is magnified by a lack of clarity in reimbursement because how payers will cover biosimilars remains unclear and new entrants often have delays in reimbursement pending specific J-code assignment.

\section{Defaults}

Defaults are essentially biases of the "status quo"; they highlight the fear of change and the uncertainty that follows. Biosimilars represent a new entrant that fundamentally challenges the current state and attempts to remove an "incumbent" biologic. Although innovation is rapidly changing how cancers are treated, the process by which oncologists decide when and how to prescribe therapy has remained the same. Decisions on what to dispense have depended on randomized clinical trial data regarding efficacy and toxicity. Not only do biosimilars challenge entrenched prescribing behaviors, but also their clinical testing is much less robust with label indications assigned by extrapolation.

\section{Familiarity}

Physician preference for the status quo is often influenced by past experiences, sometimes reaching an element of comfort or irrationality regarding a given disease or drug. Witnessing the death of a patient due to a drug's rare adverse event might cripple a physician's comfort in prescribing that drug again. Similarly, comfort with a drug that is perceived as effective and safe may be an impediment to change.

\section{Outcome Bias}

The previously described biases may be further confounded by "outcome bias," in which oncologists have grown to understand and predict responses and expected outcomes when using reference biologics. The fear that these outcomes might be jeopardized by biosimilars represents a true barrier. This behavioral challenge was partially why legislators proposed automated interchangeability - to overcome some of these difficulties. However, this law has been contested, and interchangeability is not currently allowed in the United States. ${ }^{3}$ The designation "biosimilar" in itself may magnify outcome bias because it implies that these drugs are "similar" to the reference products but not the same. In fact, our research revealed that only $69 \%$ of oncologists believe that a biosimilar has similar efficacy to a biologic, $62 \%$ agreed that the safety is equivalent, and only $12 \%$ understood the rules and regulations of extrapolation. ${ }^{8}$

\section{Availability}

Availability bias is one in which people judge the frequency of an event by the ease with which instances or occurrences can be recalled. What might be called an irrational exuberance for the more humane branded biologics after decades of witnessing the toxicities of chemotherapy might predispose to more biologics use. On the contrary, 
however, remembering when a generic drug did not elicit a response while categorizing generics and biosimilars similarly may impede prescribing.

\section{Framing}

Framing is a cognitive bias whereby humans, when making decisions, rely heavily on how the information is presented. Presenting identical information differently could lead to divergent decisions. ${ }^{9}$ Explaining to oncologists that "biosimilars are not inferior to biologics in terms of efficacy" may yield worse acceptance than stating "biosimilars are as safe and effective as their reference biologics." This is analogous to the example given by Kahneman, ${ }^{10}$ in suggesting that "odds of survival one month after surgery are $90 \%$ " is more reassuring than saying "mortality within one month of surgery is $10 \% . " 10$

\section{Anchoring}

The final bias that plays a role is the anchoring effect, which occurs when people consider a value for an unknown quantity before estimating that quantity. Oncologists might give biosimilars an impact and effect value, even before reviewing and analyzing data. This preconceived value represents an "anchor point" where subsequent information is usually formed and is the point of interpretation. Different starting points, or anchors, can yield different results and lead to diverse decision-making. ${ }^{5}$ Adjustment is a deliberate attempt to move away from the anchor, and is what oncologists must do as more data are provided so that a rational decision can be formed. An anchor point for biosimilars might be that they are not as good or effective, making the adjustment process more challenging and, to some, counterintuitive.

\section{Conclusions}

As more treatment entrants change the landscape of healthcare delivery and cost, understanding what drives prescribing habits is critical for predicting market impact. Although clinical trial data are the cornerstone for drug approval and initial market uptake, recognizing the impact of behavioral economics on why and when new entrants are dispensed is essential to developing successful strategies, especially in the biosimilars market, that hold promise for mitigating healthcare cost and upward expenditures.

\section{References}

1. Sheingold S, Nguyen NX. Impacts of generic competition and benefit management practices on spending for prescription drugs: evidence from Medicare's Part D benefit. Medicare Medicaid Res Rev 2014;4.

2. Hakim A, Ross JS. Obstacles to the adoption of biosimilars for chronic diseases. JAMA 2017;317:2163-2164.

3. Weise M, Bielsky MC, De Smet K, et al. Biosimilars: what clinicians should know. Blood 2012;120:5111-5117.

4. Weise M, Kurki P, Wolff-Holz E, et al. Biosimilars: the science of extrapolation. Blood 2014;124:3191-3196.

5. Tversky A, Kahneman D. Judgment under uncertainty: heuristics and biases. Science 1974;185:1124-1131.

6. Khullar D, Chokshi DA, Kocher R, et al. Behavioral economics and physician compensation-promise and challenges. N Engl J Med 2015;372:2281-2283.

7. Skinner JS, Volpp KG. Replacing the Affordable Care Act: lessons from behavioral economics. JAMA 2017;317:1951-1952.

8. Nabhan C, Fillman J, Ernst FR, Feinberg BA. Community oncologists' perception and understanding of biosimilars' role in oncology. Presented at the ISPOR 22nd Annual International Meeting; May 20-24, 2017; Boston, MA. Available at: https://www.ispor.org/ScientificPresentationsDatabase/Presentation/71071. Accessed November 27, 2017.

9. Tversky A, Kahneman D. The framing of decisions and the psychology of choice. Science 1981;211:453-458.

10. Kahneman D. Thinking Fast and Slow. New York, NY: Farrar, Straus, and Giroux; 2011. 